\title{
Latest Results from the Pierre Auger Observatory
}

\section{Olivier Deligny $^{* a}$ for the Pierre Auger Collaboration ${ }^{b}$}

${ }^{a}$ Institut de Physique Nucléaire, CNRS-IN2P3, Univ. Paris-Sud, Université Paris-Saclay, 91406

Orsay Cedex, France

${ }^{b}$ Observatorio Pierre Auger, Av. San Martín Norte 304, 5613 Malargüe, Argentina

E-mail: auger_spokespersons@fnal.gov

Full author list: http://www.auger.org/archive/authors_2016_11.html [www.auger.org]

The Pierre Auger Observatory, located in Argentina, has been detecting ultra-high energy cosmic rays for more than ten years. An essential feature of the $3000 \mathrm{~km}^{2}$ Observatory is its hybrid design: cosmic rays above $\simeq 10^{17} \mathrm{eV}$ are detected through the observation of the extensive air showers they induce in the atmosphere with complementary techniques, from surface detector arrays and fluorescence telescopes to radio antennas. The analyses of the multi-detector data have enabled high-statistics and high-precision studies of the energy spectrum, mass composition and distribution of arrival directions of ultra-high energy cosmic rays (UHECRs), which are the main observables to study the origin of these particles. The data have also enabled a series of analyses complementary to the primary objectives, from the search for ultra-high energy photons and neutrinos to the measurement of the proton-air cross section at $57 \mathrm{TeV}$ center-of-mass energy. A coherent interpretation of all these recent results, requiring the use of LHC-tuned interaction models to describe the air showers, opens new directions regarding the properties of UHECR sources. The resulting picture, calling into question the perception of UHECRs at the time of the conception of the Observatory in the early 1990s, has led to a plan for an upgrade of the Observatory.

38th International Conference on High Energy Physics

3-10 August 2016

Chicago, USA

\footnotetext{
* Speaker.
} 

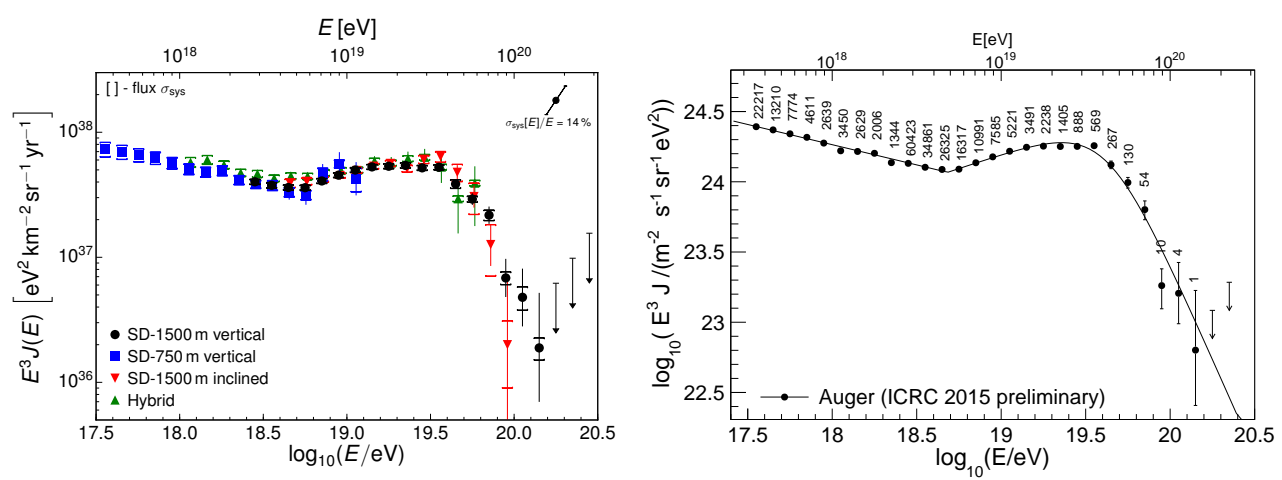

Figure 1: Left: The four energy spectra derived from SD and hybrid data. Right: The combined energy spectrum. As in the left panel, only statistical uncertainties are shown and the upper limits correspond to $84 \%$ confidence level (C.L.) [2].

UHECRs are the most energetic particles produced in nature, with energies observed up to a few times $10^{20} \mathrm{eV}$. The determination of the origin of these particles is a difficult task, mostly because of the very small value of their flux at Earth together with the fact that they experience magnetic deflections during propagation. The Pierre Auger Observatory, located in the province of Mendoza (Argentina) and covering $3000 \mathrm{~km}^{2}$, is the present flagship experiment studying UHECRs [1]. Two techniques of detection are combined to measure the extensive air shower (EAS) properties by observing their longitudinal development in the atmosphere as well as their lateral spread at ground level. Charged particles and photons that reach the ground are detected with the surface detector (SD) consisting of 1660 autonomously operated water-Cherenkov detectors. These detectors are arranged on a triangular grid of $1500 \mathrm{~m}$ spacing (the SD-1500 m array), except for a denser infill area of $\simeq 30 \mathrm{~km}^{2}$, where the spacing is $750 \mathrm{~m}$ (the SD-750 m array). A large collecting area is provided by the SD array operating 24 hours per day. The atmosphere above the SD arrays is observed by the fluorescence detector (FD) which consists of 27 fluorescence telescopes to detect the faint UV light emitted by nitrogen molecules previously excited by the charged particles from the EAS. This technique provides the opportunity of performing shower calorimetry by mapping the ionization content along the shower tracks, and of measuring the primary energy on a nearly model-independent basis. The field of view (FoV) of each telescope is $30^{\circ}$ in azimuth, and $1.5-30^{\circ}$ in elevation, except for three of them, the High Elevation Auger Telescopes (HEAT), whose $\mathrm{FoV}$ is $30-60^{\circ}$ in elevation, allowing the observation of nearby low-energy showers above the denser infill area. The FD can only operate during dark, moonless nights with a field of view free of clouds (duty cycle $\simeq 15 \%$ ). Online and long-term performances of the detectors and data quality are monitored continuously, and a set of high-quality devices installed in the Observatory array monitor the atmospheric conditions during operation. In addition, a $17 \mathrm{~km}^{2}$ sub-array of 153 radio sensors (Auger Engineering Radio Array) is dedicated to EAS radio detection.

The measurement of the flux of UHECRs above $3 \times 10^{17} \mathrm{eV}$ is emblematic of the power of using multiple detectors: it has been performed by combining four independent spectra (see figure 1, left) from the two different SD arrays (and two data sets from the SD-1500 m array, with zenith angles below $60^{\circ}$ for the vertical set and between $60^{\circ}$ and $80^{\circ}$ for the horizontal set) and from hybrid events (events detected simultaneously by the SD-1500 m array and the FD). The different 

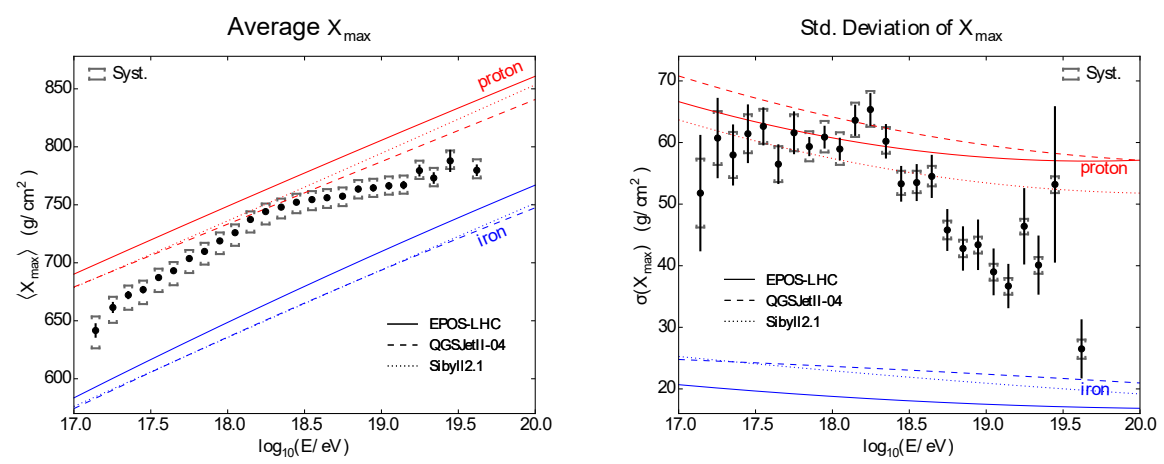

Figure 2: The mean (left) and the standard deviation (right) of the measured $X_{\max }$ distributions as a function of energy compared to air-shower simulations for proton and iron primaries [3].

data streams are complementary: data from the SD-750 m array allow for the determination of the energy spectrum down to $3 \times 10^{17} \mathrm{eV}$; data with vertical events from the SD-1500 m array are crucial above the energy of full efficiency of $3 \times 10^{18} \mathrm{eV}$ up to the highest energies, with horizontal events contributing above $4 \times 10^{18} \mathrm{eV}$ and providing an independent measurement in this energy range; and hybrid data bridge those from the two SD arrays, between $10^{18} \mathrm{eV}$ and $4 \times 10^{19} \mathrm{eV}$. The four spectra, in agreement within uncertainties, are combined into a unique one shown in figure 1, right panel, taking into account the systematics of the individual measurements. Two spectral features are established beyond doubt: the hardening in the spectrum at about $5 \times 10^{18} \mathrm{eV}$ (the so-called ankle), and a strong suppression of the flux at the highest energies, starting at about $4 \times 10^{19} \mathrm{eV}$.

The measurement of the depth of the shower maximum, $X_{\max }$, is currently the most robust mass-sensitive EAS observable. This measurement, relying on hybrid data, can be performed at the Observatory down to $10^{17} \mathrm{eV}$ thanks to the inclusion of data from the HEAT telescopes. More than 18000 events collected by the standard FD telescopes above $\simeq 6 \times 10^{17} \mathrm{eV}$, have been supplemented by 5500 events collected with HEAT. While $X_{\max }$ data cover the region of the ankle well, they do not extend into the region of flux suppression due to the intrinsically limited duty cycle of the FD. The first two moments of the $X_{\max }$ distributions, $\left\langle X_{\max }\right\rangle$ and $\sigma\left(X_{\max }\right)$, are shown in figure 2 from $10^{17} \mathrm{eV}$ to $\simeq 4 \times 10^{19} \mathrm{eV}$. Data are compared to simulations, for proton and iron primaries, performed using three hadronic interaction models that were either tuned to recent LHC data (QGSJetII-04 [4], EPOS-LHC [5]) or found in good agreement with LHC measurements (Sibyll2.1 [6]). Between $10^{17} \mathrm{eV}$ and $2 \times 10^{18} \mathrm{eV},\left\langle X_{\max }\right\rangle$ increases by around $85 \mathrm{~g} \mathrm{~cm}^{-2}$ per decade of energy. This is larger than that expected for a constant mass composition $\left(60 \mathrm{~g} \mathrm{~cm}^{-2}\right.$ per decade) and thus indicates that the mean primary mass is becoming lighter over this energy range. On the other hand, above $2 \times 10^{18} \mathrm{eV}$, the rate of change of $\left\langle X_{\max }\right\rangle$ becomes significantly smaller $\left(26 \mathrm{~g} \mathrm{~cm}^{-2}\right.$ per decade). The trend is thus inverted and the composition gets heavier. The fluctuations of $X_{\max }$ start to decrease above the same energy, $2 \times 10^{18} \mathrm{eV}$, being rather constant below. The conversion of the first two moments of the $X_{\max }$ distributions to the mean value of $\ln A$ and its variance $\sigma(\ln A)$, confirms in a quantitive way this evolution of the average composition [3].

An attempt to understand the origin of the flux suppression at the highest energies has been conducted by simultaneously fitting both the spectrum and the evolution of $X_{\max }$ data towards the 

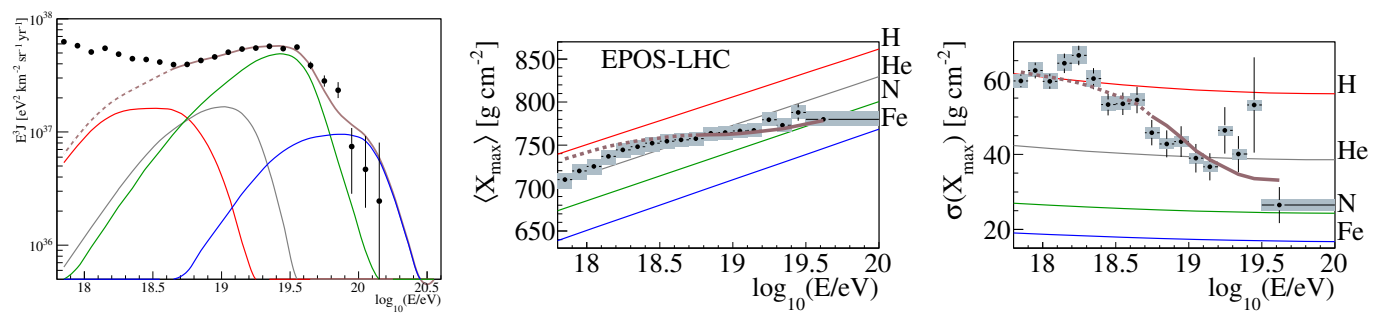

Figure 3: Left: Energy spectrum of UHECRs with the best-fit elemental contributions (see text). Center and right: Resulting average and standard deviation of the $X_{\max }$ distributions (assuming EPOS-LHC for the interactions) for the model prediction (brown), pure ${ }^{1} \mathrm{H}$ (red), ${ }^{4} \mathrm{He}$ (grey), ${ }^{14} \mathrm{~N}$ (green) and ${ }^{56} \mathrm{Fe}$ (blue). Only the energy range where the brown lines are solid is included in the fit [7].

highest energies, above $5 \times 10^{18} \mathrm{eV}$, where UHECRs are presumably of extragalactic origin. In the assumed astrophysical benchmark scenario, identical sources homogeneously distributed in a comoving volume and injecting ${ }^{1} \mathrm{H},{ }^{4} \mathrm{He},{ }^{14} \mathrm{~N}$ and ${ }^{56} \mathrm{Fe}$ nuclei are considered. For each species $i$, a power-law energy spectrum $Q_{i}\left(E_{\text {inj }}\right)$ is assumed with a broken exponential rigidity cutoff $R_{\text {cut }}, Q_{i}\left(E_{\text {inj }}\right)=Q_{0} p_{i}\left(E_{\text {inj }} / \mathrm{EeV}\right)^{-\gamma}$ for $E_{\text {inj }} \leq Z_{i} R_{\text {cut }}$ and $Q_{0} p_{i}\left(E_{\text {inj }} / E e V\right)^{-\gamma} \exp \left(1-E_{\text {inj }} / Z_{i} R_{\text {cut }}\right)$ for $E_{\text {inj }} \geq Z_{i} R_{\text {cut }}$. The free parameters of the fit are the normalization constant $Q_{0}$, the spectral parameters $\gamma$ and $R_{\text {cut }}$, and three of the mass fractions $p_{i}$ (the fourth being bound by $\sum_{i} p_{i}=1$ ). Cosmic rays are propagated from the sources to the observer accounting for the various interactions with the encountered photon backgrounds. The simulated spectra and the mean and variance of the simulated $X_{\max }$ distributions corresponding to the best fit are shown in figure 3. The spectrum is best fitted by a succession of cutoffs of the different groups of elements, with $R_{\text {cut }} \simeq 4.6 \times 10^{18} \mathrm{~V}$, thus pointing to the flux at Earth being partly limited by the maximum energy at the source. The best fit returns $\gamma \simeq 1$, suggesting a very hard source spectrum, and an injection of mostly intermediate mass nuclei, with very few protons or iron nuclei. Note that the goodness of the fit is sensitive to systematic uncertainties in the photodisintegration rates, the hadronic interaction models used to infer $X_{\max }$ properties of each observed primary species, and the absolute energy scale of the Observatory. Considering more injection masses, non-uniform source distributions, or more complicated injection spectra, could also improve the agreement between the benchmark scenario and the data.

The energy losses of UHECRs limit the horizon of the highest-energy particles. For smallenough magnetic deflections, the distribution of the arrival directions of UHECRs above $\simeq 4 \times$ $10^{19} \mathrm{eV}$ could mirror the inhomogeneous distribution of the nearby extragalactic matter. The search for anisotropy at small and intermediate angular scale at the highest energies is thus potentially the most powerful way to infer the sources of UHECRs. Data have been subjected to comprehensive anisotropy searches for different energy thresholds between $4 \times 10^{19} \mathrm{eV}$ and $8 \times 10^{19} \mathrm{eV}$, and within different angular windows, between $1^{\circ}$ and $30^{\circ}$ [8]. Searches for significant excesses anywhere in the sky have been performed, as well as searches for correlations with known astrophysical structures and with objects that are considered plausible candidates for UHECR sources. Out of all the searches performed, none of the analyses provides any statistically significant evidence of anisotropy. The two largest departures from isotropy, both with post-trial probability $\simeq 1.4 \%$, are found for energies in excess of $5.8 \times 10^{19} \mathrm{eV}$ when looking, on the one hand, within $15^{\circ}$ of the direction of Centaurus A - the closest radio-loud active galactic nucleus (AGN), and when looking, 

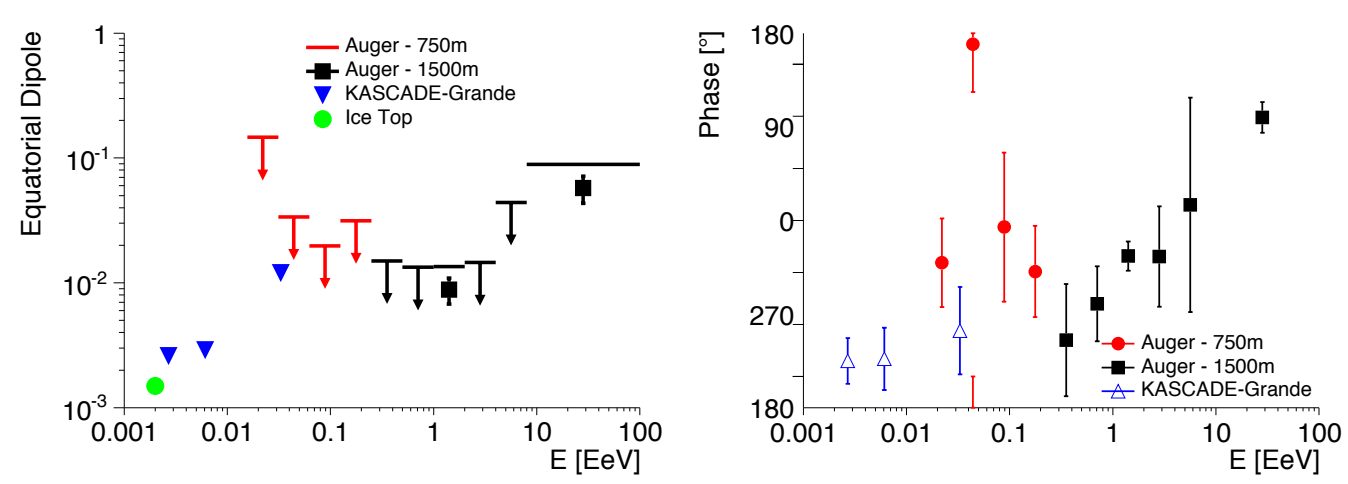

Figure 4: Left: Upper limits to the dipole equatorial component at the 99\% C.L.. Amplitudes are also reported (black squares) in the two energy bins when the corresponding $p$-value expected from isotropy is below $10^{-3}$ (see text). Right: Phases of the first harmonic modulation in RA [10].

on the other hand, within $12^{\circ}$ of the objects collected in the flux-limited catalog of AGNs observed in X-rays (Swift-BAT-70 [9]) closer than $130 \mathrm{Mpc}$ and brighter than $10^{44} \mathrm{erg} / \mathrm{s}$.

Large-scale anisotropies are possible signatures of a collective motion of cosmic rays and/or of the global distribution of their sources at all energies. The dipole moment is of special interest, and various techniques can be used to infer the equatorial component of the dipole even for energies well below the full efficiency. As in none of the energy bins are the $p$-values for the amplitudes of the first harmonic at the level of discovery, the upper limits, at the $99 \%$ C.L., to the equatorial component of the dipole are shown in the left panel of figure 4 as a function of energy. Amplitudes are also shown in the two energy bins where the $p$-values are $1.5 \times 10^{-4}$ and $6.4 \times 10^{-5}$. The corresponding phases are shown in the right panel as a function of energy. Interestingly, the phase above $8 \times 10^{18} \mathrm{eV}, \simeq 95^{\circ}$ in right ascension, is roughly the opposite of the one at energies below $10^{18} \mathrm{eV}$, which is in the general direction of the Galactic center. The percent limits to the amplitude of the anisotropy exclude the presence of a large fraction of Galactic protons at EeV energies [11]. Accounting for the inference from $X_{\max }$ data that protons are in fact abundant at those energies, this might indicate that this component is extragalactic, gradually taking over a Galactic one. The low level of anisotropy would then be the sum of two vectors with opposite directions, naturally reducing the amplitudes. This scenario is to be explored with additional data.

The reach of the data of the Observatory goes beyond the study of UHECRs. Among others, the measurement of the p-air cross-section is an illustration of the capability of the Observatory to address particle interactions. The technique relies on the analysis of the shape of the distribution of the largest measured values of $X_{\max }$. The tail of this distribution is directly related to the distribution of the first-interaction point of the primary cosmic ray, which is inversely proportional to the p-air cross-section. The observable is the slope of the exponential tail, $\Lambda_{\eta}$, which is a measure of the shower attenuation length in atmosphere. Using $\eta=20 \%$ for the fraction of the most deeply penetrating showers in two energy ranges around $10^{18} \mathrm{eV}$ where the shape of the $X_{\max }$ distribution is compatible with the presence of a fraction of protons greater than $20 \%$, the measured values of $\Lambda_{\eta}$ are $60.7 \pm 2.1 \pm 1.6 \mathrm{~g} / \mathrm{cm}^{2}$ and $57.4 \pm 1.8 \pm 1.6 \mathrm{~g} / \mathrm{cm}^{2}$ in the two energy bins, respectively, corresponding to center-of-mass energies of 38.7 and $55.5 \mathrm{TeV}$. The value of $\sigma_{\mathrm{p}-\text { air }}$ is then derived from Monte-Carlo simulations using the most recent hadronic models. The two measurements are 


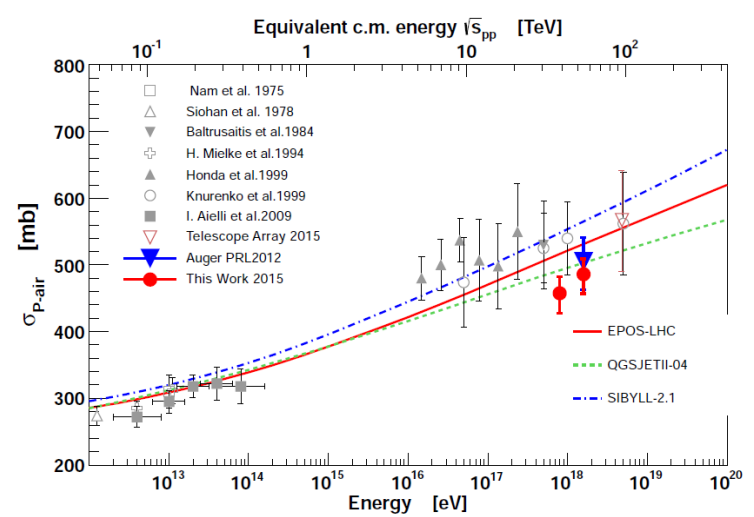

Figure 5: The $\sigma_{\mathrm{p}-\text { air }}$ measurement compared to previous data and model predictions [12].

shown in figure 5 compared to the previous measurements and to model predictions. The data are consistent with a cross-section rising with energy; however, the statistical precision is not yet sufficient to make a statement on the functional form.

The huge collecting area and the hybrid detection mode of the Observatory have yielded important advances in the measurements of UHECRs and challenged the traditional literature. However, the mass composition of UHECRs into the suppression region remains unexplored due to the limited duty cycle of the FD. It follows that equipping the SD to assess mass composition at the highest energies is the natural evolution of the Observatory. The installation of a plane of $4 \mathrm{~m}^{2}$ plastic scintillators above each station will provide additional measurements of the showers helping to produce composition-sensitive observables in an energy range including the highest energies, and will open the possibility to search for a small fraction of light primaries at the highest energies [13]. The operation of such an upgraded array is anticipated between 2018 and 2024.

\section{References}

[1] The Pierre Auger Collaboration, Nucl. Instrum. Meth. A 798 (2015) 172

[2] I. Valino for the Pierre Auger Collaboration, Proc. 34th ICRC, (2015) arXiv:1509.03732

[3] A. Porcelli for the Pierre Auger Collaboration, Proc. 34th ICRC, (2015) arXiv:1509.03732

[4] S. Ostapchenko, Phys. Rev. D, 83 (2011) 014018

[5] T. Pierog and K. Werner, Phys. Rev. Lett., 101 (2008) 171101

[6] E. J. Ahn et al., Phys. Rev. D, 80 (2009) 094003

[7] A. Di Matteo for the Pierre Auger Collaboration, Proc. 34th ICRC, (2015) arXiv:1509.03732

[8] The Pierre Auger Collaboration, ApJ 804 (2015) 15

[9] W.H. Baumgartner et al., ApJ 207 (2013) 19

[10] I. Al Samarai for the Pierre Auger Collaboration, Proc. 34th ICRC, (2015) arXiv:1509.03732

[11] The Pierre Auger Collaboration, ApJS 203 (2012) 34

[12] R. Ulrich for the Pierre Auger Collaboration, Proc. 34th ICRC, (2015) arXiv:1509.03732

[13] The Pierre Auger Collaboration, arXiv:1604.03637 